\title{
Generating Facial Composites from Principal Components
}

\author{
Barbora Zahradnikova ${ }^{1}$, Soňa Duchovicova ${ }^{1}$ and Peter Schreiber ${ }^{1}$ \\ ${ }^{1}$ Institute of Applied Informatics, Automation and Mechatronics, Faculty of Materials Science and Technology, Slovak University of \\ Technology, 91701 Trnava, Slovakia
}

\begin{abstract}
Penetrating computers and process automation into all the fields of human activity meant a breakthrough in the research of forensic methods applied on facial composite production. Although the issues stemming from unsatisfying graphical presentation of composites have been resolved by implementing computational technique into creating facial composites, software developed and used by the police in the most countries of the world still has not achieved satisfactory results. The research presented in this paper aims at developing an alternative technique respecting human ability of recognizing faces and eliminating the limitations of the previous systems as identified by psychologists within several years. The paper is concerned with a novel method of generating facial pictures. We presented the initial research on holistic and hybrid model and demonstrated the ability of generating novel facial images based on the proposed solution.
\end{abstract}

\section{Introduction}

Facial composites are pictures of suspects, usually applied in criminal investigation. Traditionally, facial composites are created by a police artist according to a description of the witness. Producing facial likenesses has been an issue for almost a hundred years especially in the fields of criminology and psychology.

Although penetrating computational technique into the field solved many issues stemming from often unsatisfying graphical representation of manually drawn or mechanically created composites, the naming rate when applying facial composite technique in personal identification has been only slightly improved. Utilising the currently available software (such as Identi-Kit, Photo-Fit, E-Fit, Mac-a-Mug or Faces) leads to naming rates reaching only up to $17 \%$ in laboratory conditions (with a photograph present during composite production) and only $1-2 \%$ in real conditions [1][2][3][4].

Several psychological surveys indicate that the main problem does not lie in the graphics. Instead, the low results are reached due to the whole process of identification, mainly on the way a witness is asked to describe a suspect. From the psychological point of view, the procedure of identification consists of two independent processes: recall of the target face (the task of a witness) and recognition of the target face (identifying the person based on the portrait).

Traditionally, a witness is required to describe a suspect in terms of individual features, or to choose the features from a database. In this way, the witness is forced to imagine a suspect and decompose the face in the mind. This kind of recalling information from the memory is a process not compatible with the way humans perceive other faces, as described e.g. by [5][6]. Faces are claimed to be viewed, stored and retrieved at a holistic level. The faces are believed to be processed as a whole also by [7], including their similarities to each other.

In this paper, a method of holistic representation of faces is presented as a first step in developing a system relying on the human ability of recognising faces. The authors describe the possible approaches of producing holistic facial images and demonstrate the ability of generating facial image that could be presented to a witnesses for the identification based on the proposed solution. By presenting the witness with whole faces, witness is not forced to recall and decompose the face of a suspect.

\section{System description}

The presented solution relies on utilising the human ability of recognising faces. Instead of forcing a witness to imagine a face and decompose it into individual features, the introduced method provides a witness with a series of facial images. The witness does not need to select the appropriate nose, mouth or eyes. Instead, the face most resembling the suspect should be named. This approach is similar to identifying criminals from the lineup of suspects.

Based on the selection, optimization method is to be deployed and new faces are generated. In each iteration, witness is supposed to choose the best match and new faces are evolved accordingly. The optimization method is still under development. More information can be found in [8] 


\subsection{Facial model}

A sketch-like system based on recombining and muting individual features was proposed and implemented in the previous research [8]. The system (see Figure 1) proved the hypothesis of converging to a stable solution. However the time and memory consumption was not satisfying requiring approximately 5 minutes for creating each generation. Presuming the future system based on features extracted from photographs, this solution cannot be considered to be the optimal one.

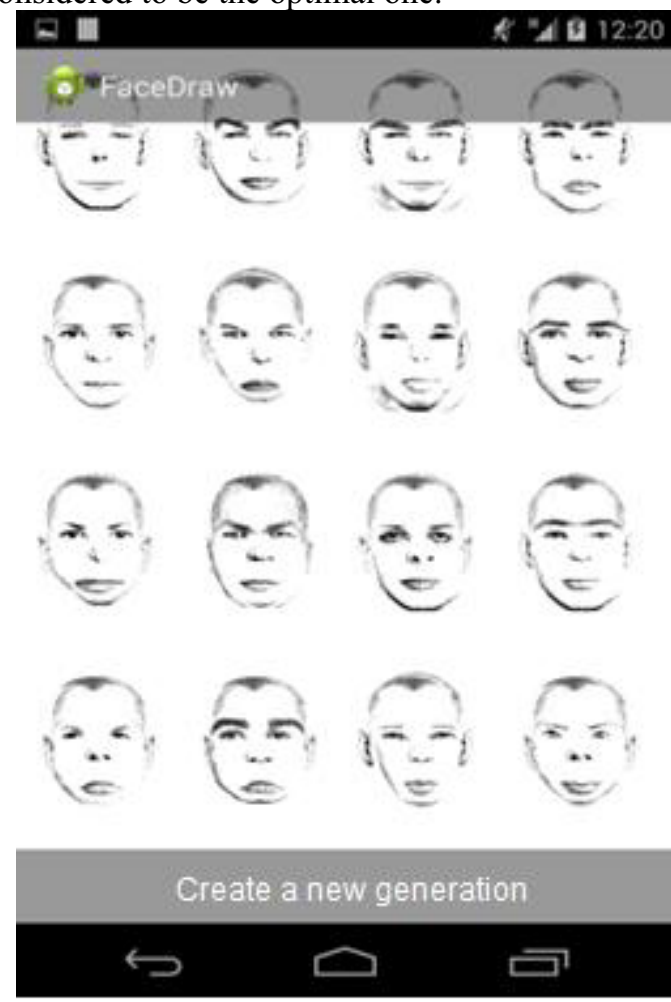

Figure 1Pilot version of the system

The pilot version of the system utilised individual facial features as inputs for generating a face. These were further bred in order to produce novel faces. Due to the proposed model of face, the system was forced to work with a huge amount of images, which was a strategy both time and memory consuming.

In effort to provide a more feasible solution, the authors decided to implement a holistic model of face.

\subsubsection{Holistic Approach}

Holistic approach, as mentioned above, stands for methods utilising whole faces as input data. The methods are based on the similarity of faces expressed by statistical regularities. These techniques are usually based on the Principal Component Analysis, a technique enabling reducing the dimensionality of data space to the smaller dimensionality of feature space by extracting the major axis of variation in a data set [9].

The model was derived by processing 87 (M) 2-D greyscale images. Before the PCA was applied, each 2-D image was transformed to a $1-D$ vector by concatenating the rows into a long vector. In this way, 87 vectors of size $\mathrm{n}$ (columns $\mathrm{x}$ rows) were obtained.

$$
x_{i}=\left[p_{1}, p_{2} . . p_{n}\right]^{T}
$$

where ${ }^{i}$ stands for the image number and $p$ represents a pixel within the vector.

Based on the $M$ vectors, the mean image calculated as:

$$
m=\frac{1}{M} \sum_{i=1}^{M} x_{i}
$$

and extracted from each image:

$$
w_{i}=x_{i}-m
$$

in order to get the matrix $W$ consisting of the mean centred images $w_{i}$.

$$
C=W^{T} \cdot W
$$

However, as computing the covariance matrix can be very time and memory consuming, matrix $L$ linearly related to $C$ was calculated instead:

$$
L=W \cdot W^{T}
$$

Followingly, based on the matrix $L$, the eigenvectors $(V)$ and eigenvalues $(D)$ were obtained so that:

$$
x=\bar{x}+P \cdot b
$$

where $P$ consists of eigenvectors of the covariance matrix ( $P=W^{T} \cdot V$ ) and $b$ represents the coefficients used to reconstruct a face.

Eigenvectors (eigenfaces) and eigenvalues are the principal components of an image and describe the relation of an eigenface to the mean image. They can be understood as a measure of similarity to the mean image. The mathematical background of eigenvectors calculating can be found in [10].

\subsubsection{Active Shape Model}

The role certain human features (especially eyes and mouth) play in perceiving faces cannot be denied. Additionally, when the features are not specified and images are recombined as wholes, the features tend to appear blurred. A method to overcome the issues arising when holistic techniques are applied on face recognition was outlined by [11]. It is based on separating the shape and texture information in order to ensure sharper solutions.

The hybrid method differs from the classical holistic technique in the way the face is decomposed into principal components.

Prior to applying the PCA, the shape information consisting of $87 x, y$ components was extracted from each face and transformed to $1-\mathrm{D}$ vectors $y_{i}$ by concatenating the $x$ and the $y$ components. The matrix $Y$ having the dimension of $174 \times 87(q \times M)$ was obtained.

$$
y_{i}=\left[p_{1}, p_{2} . p_{q}\right]^{T}
$$

Followingly, the reference shape was counted according to the formula: 


$$
Y_{\text {std }}=\frac{1}{M} \sum_{i=1}^{M} y_{i}
$$

Afterwards, all the images were warped into the reference (average) shape applying the Delaunay triangulation and generated a set of textures [12] .

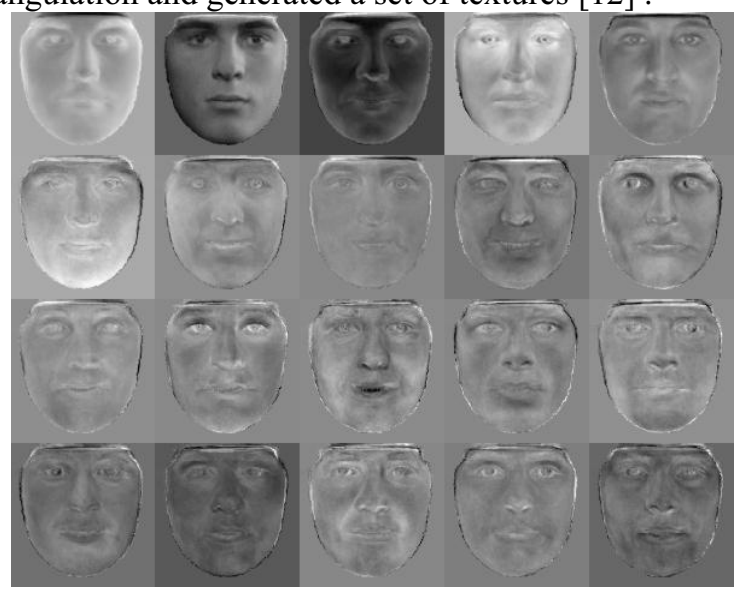

Figure 2 Visualising of texture eigenfaces

After reshaping, normalising and warping the images to the reference shape, the average texture was counted, similarly as in (2) and (8).

Followingly, the Principal Component Analysis was performed on shapes and textures separately, proceeding according to (4), (5) and (6) and obtaining the texture (visualised in Figure 2 ) and shape eigenfaces.

\subsection{Optimization of the image}

Following the aim of simplifying the process of creating the facial composite, generating the images of facial likeness utilising a genetic algorithm (graphically depicted in Figure 3) was proposed. The procedure should be both, more comfortable for a forensic technician and at the same time more natural for a witness.

The objective of such a system is not to let the witness describe and compose a portrait. On the contrary, the new approach resembles choosing a perpetrator from a lineup of suspects

The witness is presented with a series of portraits and is expected to choose the one most resembling the suspect. In further steps, new faces generated applying a GA from [8] are presented until the resemblance cannot be evaluated by the witness. Then, several faces are generated randomly or the target face is to be chosen.

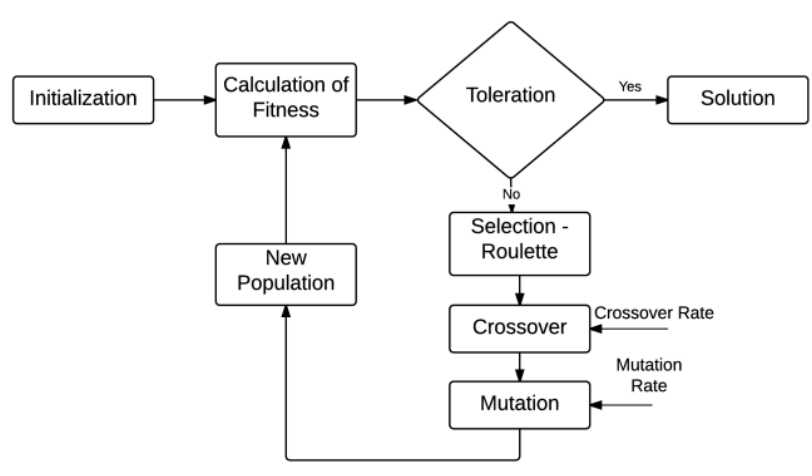

\section{Figure 3 Proposal of a Genetic Algorithm}

For the real implementation, applying interactive genetic algorithm is a prerequisite as only the witness can evaluate the resemblance between the generated images and the suspect. However, in order to make evaluating the applicability of the proposed model easier, we utilised a computerised method based on automated, mathematically calculated evaluation.

\section{Implementation}

The system as described in the previous section was implemented using Matlab.

At the beginning, models of face were derived using 87 images and 87 landmark files corresponding to the images. The authors created a holistic model as well as Active Shape Model and tested their usability for the purposes of the system automatically generating facial composites.

Consequently, the principal components of models were recombined and the images were reconstructed using newly obtained coefficents. Comparing the methods in both time consumption and final image sharpness were used as parameters for comparing the appropriateness of the tested methods.

Interactive genetic algorithm was not used for the evaluation despite the fact it is the most reliable measure for testing the system as a whole. As only suitability of the mathematical models of a face was to be tested, implementing mathematically calculated evaluation helped increasing the effectiveness and reducing the time necessary to carry out the tests.

As a measure for evaluating the individuals obtained applying a GA, fitness function is used. The fitness represents the similarity of individual faces with respect to the target face. Each novel face is to be compared to the target face and based on the received ranking, parents for crossover are to be selected.

Root mean squared error was used as the main metric for evaluating the similarity between the generated composites and a target image. Compared to Euclidean distance, Mahalanobis Distance and Angle metric, the RMSE was evaluated as the best match if similarity of faces is to be considered [13].

The RMSE is calculated as follows:

$$
\text { Fittness }=\sqrt{\frac{1}{n} \sum_{i=1}^{n}\left(x_{i}-x_{t \arg e t}\right)^{2}}
$$




\section{Results and Discussion}

The goal of testing was to reconstruct an image from the original database, as well as to build a novel face using random combination of face coefficients applying both holistic model and Active Shape Model.

A number of practical advantages of the holistic approach were described in literature [14]. Firstly, locating facial features is not required since the face is handled as a global feature. Secondly, the image resolution can be relatively low. Thirdly, the low resolution is responsible for reducing the computational load which proved correct during the testing. However, despite the presented advantages, the ability of deriving new faces from the holistic model is only restricted.
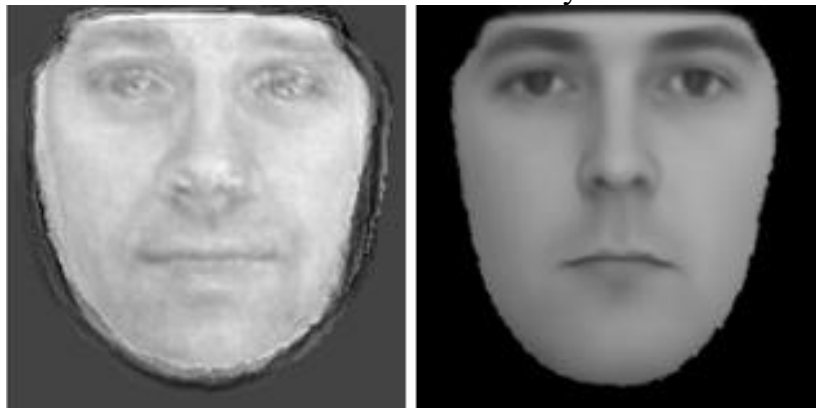

Figure 4a Random image - Holistic Model (left) Figure 4b Random image - Acive Shape Model (right)

When the further breeding was applied on the principal components retrieved from whole faces/images, the faces generated were rather blurred (Figure 4a). Therefore, utilising exclusively the holistic approach for the whole face does not appear appropriate in this case. However, reduction in time and memory consumption in comparison with using individual facial features is remarkable requiring less than 30 seconds per generation.

Applying the ASM resulted in much sharper images (Figure 4b). It both preserves the psychologically important information concerning the individual facial features and provides satisfactory solution at acceptable time (less than 1 minute per generation) and memory consumption.

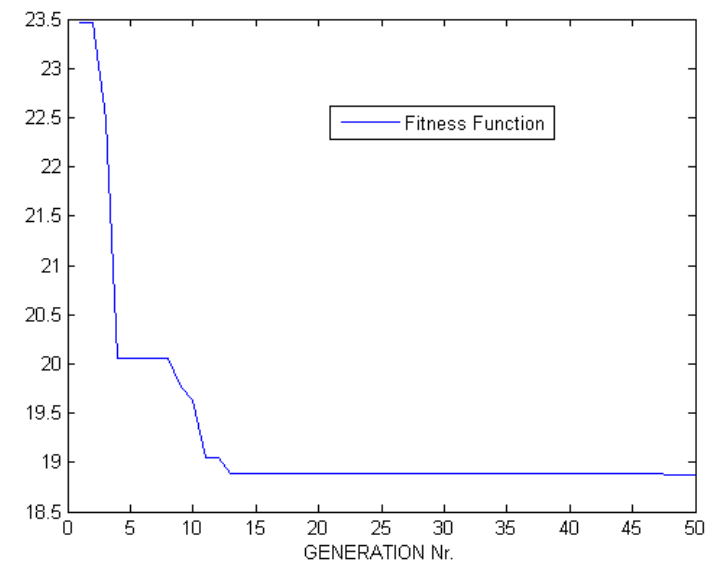

Figure 5 Fitness Function progress within 50 generations
The testing approach additionally revealed also only restricted appropriateness of the proposed genetic algorithm as an optimization method. As the obtained final RMSE has not reached satisfactory values (see the final image in Figure 6 and the slow convergence rate of Fitness function depicted in Figure 5).

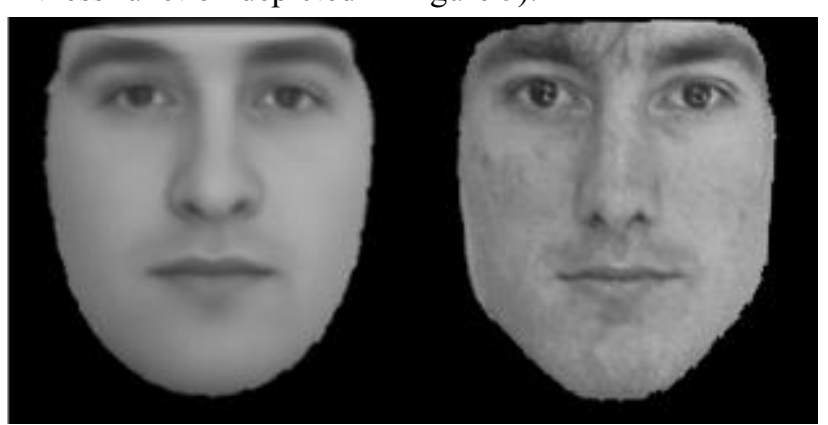

Figure 6 Final Image (after 50 generations) left, target face right

\section{Conclusion}

New, holistic method of generating a novel facial picture was proposed and tested. The technique could be applied in a system automatically generating facial composites enabling an easier and more natural process of creating "identikit images".

Active Shape Model proved to be more appropriate for the described purpose as it is both able to preserve the psychologically important information concerning the individual facial features and capable of producing accurate and sharp results at acceptable time.

As the presented solution is a pilot project, the authors concentrate on the visual likeness of internal region of face, not including the external features such as hair, glasses, moustaches and beards. These might be implemented later, however they are not the key issues in creating a facial composite, as these elements can be easily changed.

Currently, the authors identified developing an appropriate optimization method as a key issue for the future research.

\section{Acknowledgement}

The submitted paper was supported by the project Promoting Young Researchers 2015 - Slovak University of Technology in Bratislava, No. 1340 - "Evolving Faces Using Genetic Algorithms"

This publication is the result of implementation of the project VEGA 1/0673/15: "Knowledge discovery for hierarchical control of technological and production processes" supported by the VEGA.

\section{References}

1. H. D. Ellis, "Practice aspects of face memory," in Wells, G.R., Loftus, E.F., editors, Eyewitness Testimony. Psychological Perspectives. England: Cambridge University Press, pp. 12-37, (1984) 
2. N. Brace, G. Pike and R. Kemp, "Investigating EFIT using famous faces, Forensic Psychology and Law, pp $272-276$ (2000)

3. D. McQuiston-Surrett, L. D. Topp, and R. S. Malpass. "Use of facial composite systems in US law enforcement agencies." Psychology, Crime \& Law 12.5: 505-517. (2006)

4. T. Valentine, and J. P. Davis. Forensic Facial Identification: Theory and Practice of Identification from Eyewitnesses, Composites and CCTV. John Wiley \& Sons, 2015.

5. J. W. Tanaka and M. J. Farah, "The holistic representation of faces," Perception of faces, objects, and scenes: Analytic and holistic processes, 53-74 2003

6. B. Meinhardt-Injac, M. Persike., and G. Meinhardt, "Holistic face processing is induced by shape and texture." Perception. 42, pp 716-732. doi: 10.1068/p7462 (2013)

7. H. Moon and P. J. Phillips, "Computational and performance aspects of PCA-based face-recognition algorithms," Perception. London, 30.3: 303-322 2001

8. B. Zahradnikova, S. Duchovicova and P. Schreiber, "Facial composite system using genetic algorithm," in International Doctoral Seminar 2014: Proceedings of the 9th International Doctoral Seminar (IDS 2014), Zielona Góra, Poland, May 19 21, 2014. - ISBN 978-80-8096-195-4. - S. 270-274 (2014)

9. K. Kim, "Face recognition using principle component analysis," in International Conference on Computer Vision and Pattern Recognition. p. 586591 (1996)

10. L. I. Smith, "A tutorial on principal components analysis," Cornell University, USA, 51: 52 (2002)

11. I. Craw and P. Cameron, "Face recognition by computer," in BMVC92. Springer London, p. 498507 (1992)

12. M. De Berg, C. Otfried, M. Van Kreveld and M. Overmars, "Computational Geometry: Algorithms and Applications, " Springer-Verlag, rd edition, ISBN 978-3-540-77973-5 (2008)

13. Ch. Frowd, et al. "Automating the Processes Involved in Facial Composite Production and Identification." Bio-inspired Learning and Intelligent Systems for Security, 2009. BLISS'09. Symposium on. IEEE, (2009)

14. J. H. Lai, P. C. Yuen and G. C. Feng, "Face recognition using holistic Fourier invariant features," Pattern Recognition, 34.1: 95-109 2001

15. Y. Liu, S.C. Zhu, Face data. Pattern Recognition and Machine Learning. Available at < www.stat.ucla.edu/ sczhu/Courses/UCLA/Stat_231/ Project_I/face_data.zip $>$ 